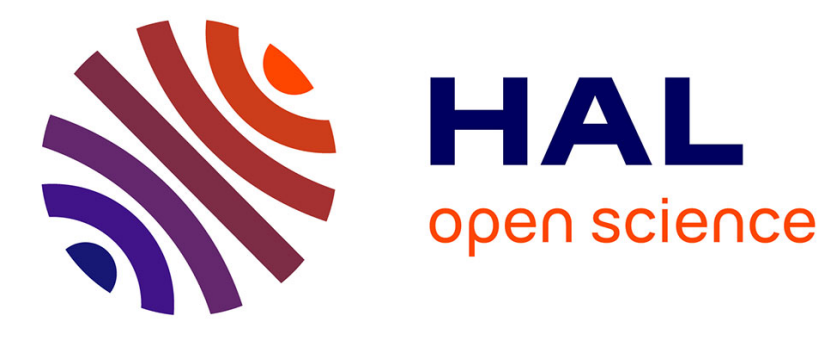

\title{
L'influence de l'épargne de précaution sur la recherche d'emploi
}

Yann Algan, Antoine Terracol

\section{To cite this version:}

Yann Algan, Antoine Terracol. L'influence de l'épargne de précaution sur la recherche d'emploi. Economie et Statistique / Economics and Statistics, 2001, 349-350, pp.63-72. hal-01017893

\section{HAL Id: hal-01017893 \\ https://hal-sciencespo.archives-ouvertes.fr/hal-01017893}

Submitted on 3 Jul 2014

HAL is a multi-disciplinary open access archive for the deposit and dissemination of scientific research documents, whether they are published or not. The documents may come from teaching and research institutions in France or abroad, or from public or private research centers.
L'archive ouverte pluridisciplinaire HAL, est destinée au dépôt et à la diffusion de documents scientifiques de niveau recherche, publiés ou non, émanant des établissements d'enseignement et de recherche français ou étrangers, des laboratoires publics ou privés. 


\title{
L'influence de l'épargne de précaution sur la recherche d'emploi
}

\author{
Yann Algan et Antoine Terracol*
}

L'étude de l'incidence des dispositifs d'assurance chômage sur l'offre de travail s'est limitée jusqu'ici, faute de données, au système de protection sociale au travers de l'allocation chômage. Cependant, au cours des années 90, de plus en plus de ménages ont constitué une épargne de précaution afin de compléter l'indemnisation partielle prévue par ce système. Pour l'une des premières fois, en réunissant des informations relatives à l'activité, au revenu, mais aussi au patrimoine des chômeurs, le Panel européen des ménages permet de mesurer l'impact de cette auto-assurance sur le comportement d'offre de travail des chômeurs.

Cette épargne de précaution permet au chômeur d'être plus sélectif dans la recherche d'un emploi : plus elle est élevée, plus le salaire minimal à partir duquel il accepte une offre d'emploi (salaire de réserve) est élevé. Les chômeurs dépourvus d'épargne restent plus longtemps au chômage que ceux qui en sont détenteurs, mais la durée de chômage est d'autant plus longue que son niveau est élevé.

Cette influence ne s'exerce toutefois de manière significative qu'au-dessus d'un patrimoine de 4600 euros (30 000 francs), montant à peu près équivalent à une année d'allocation chômage en moyenne : cette épargne de précaution n'a d'impact sur la stratégie de recherche d'emploi que pour des niveaux équivalents à ceux de l'assurance chômage. En ce qui concerne le type d'épargne, enfin, seul le livret d'épargne possède le degré de liquidité requis pour jouer le rôle d'épargne de précaution contre les risques du marché du travail. 
$\mathbf{L}$ 'incidence des mécanismes d'assurance chômage sur le comportement d'offre de travail est au cœur de la réflexion sur les systèmes de protection sociale. Mais paradoxalement, le débat s'est limité jusqu'à maintenant à l'étude des effets empiriques des allocations chômage (Mortensen, 1986). Or ces dernières ne constituent qu'une forme d'assurance chômage : les ménages peuvent avoir recours à un comportement d' « auto-assurance » en constituant une épargne de précaution. Un tel comportement est ainsi invoqué comme une composante essentielle de la hausse des taux d'épargne européens à la suite de l'aggravation des risques de chômage et de la restriction de l'accès aux allocations dans les années 90 (Villieu, 1997).

Les effets théoriques de l'épargne de précaution sur l'offre de travail ont été depuis longtemps établis par Danforth (1979). Ce dernier montre que l'épargne partage les mêmes vertus assurantielles que les allocations en autorisant un chômeur à rechercher plus longtemps la meilleure offre d'emploi possible. Il en dégage trois propositions : les riches sont plus sélectifs dans leur recherche, la durée de leur épisode de chômage est en conséquence plus longue mais le rendement de leur recherche est aussi plus élevé. Des travaux théoriques plus récents (Hansen et Imrohoroglu, 1992) exploitent cette relation pour réévaluer le niveau optimal des allocations chômage lorsque les individus peuvent également s'auto-assurer en épargnant (1)

Mais cette littérature manque encore de fondements empiriques (2). Cette absence est principalement due au manque de bases de données adéquates. L'estimation de l'impact de l'épargne de précaution nécessite en effet une base qui réunisse à la fois des calendriers d'activité, de revenu et de patrimoine des individus. Or la spécificité et la richesse du Panel européen des ménages est précisément de réunir ces informations sur une période longue, de 1993 à 1996 (3). Cette enquête répertorie quatre types d'actifs qui sont susceptibles, à des degrés divers, d'être utilisés comme épargne de précaution: les livrets d'épargne, l'épargne logement, les valeurs mobilières et les produits d'assurance-vie (cf. encadré 1). Cette base de données offre en complément des informations sur les variables susceptibles d'affecter le comportement de recherche d'emploi telles que l'âge, le sexe, le niveau de qualifications et la perception d'allocations chômage. Il est ainsi possible d'isoler les effets propres à l'épargne de précaution sur l'offre de travail des chômeurs.
Pour estimer l'impact de cette auto-assurance, on s'inspire de la littérature économétrique consacrée au rôle des allocations chômage. Cette dernière dégage deux effets principaux de l'assurance vis-à-vis du salaire de réserve et de la durée du chômage, correspondant aux deux premières propositions de Danforth (1979). On est ainsi conduit à évaluer l'impact du stock d'épargne sur le salaire minimal à partir duquel un chômeur accepte une offre d'emploi (salaire de réserve), ainsi que sur la durée de recherche d'emploi. Livret d'épargne, épargne logement, valeur mobilière et assurance-vie sont des types d'épargnes qui diffèrent par leur finalité, aussi bien que par leur degré de liquidité. Ils présentent de plus une attractivité très différenciée pour les chômeurs : l'épargne mobilière, par exemple, s'avère peu attractive et peu présente au sein de cette population. L'effet sur le salaire de réserve et la sortie du chômage varie-t-il selon le type d'épargne? Une telle influence s'exerce-t-elle quel que soit le montant de l'épargne, ou, au contraire, seulement à partir d'un certain seuil ?

\section{L'impact de l'épargne de précaution sur le salaire de réserve}

$\mathbf{O}^{\text {n }}$ estime dans un premier temps l'influence du stock d'épargne détenu par un chômeur sur son salaire de réserve. À l'instar de la plupart des études empiriques relatives au salaire de réserve, l'approche retenue s'inscrit dans la perspective théorique des modèles de prospection d'emploi initiée par McCall (1970). Dans ce cadre, la probabilité d'accepter une offre d'emploi repose sur un arbitrage intertemporel. À chaque période, un chômeur reçoit, avec une certaine probabilité, une offre d'emploi associée à un niveau donné de salaire. Il décide alors de continuer sa prospection tant que l'espérance de revenu apporté par l'emploi est inférieure au

\footnotetext{
1. Ce sujet a récemment fait l'objet d'une abondante littérature (Acemoglu et Shimer, 1998; Wang et Williamson, 2000 ; Algan et al., 2000 ; Joseph et Weitzemblum, 2000).

2. À notre connaissance, la seule étude empirique sur ce sujet est due à Stancanelli (1999) sur données anglaises.

3. Le Panel Study of Income Dynamics américain fournit des données très détaillées sur la richesse financière des agents en compléments des calendriers d'activités et de revenus. Cependant, ces derniers sont annuels alors que les informations sur la richesse sont quinquennales. II est alors impossible de suivre l'évolution conjointe de ces variables. En outre, l'enquête Suivi des chômeurs donne des renseignements sur le stock d'actif initial (voir Cases, 1996a). Mais l'information est trop peu riche pour une étude du lien entre épargne et recherche d'emploi.
} 
gain marginal espéré en attendant une offre plus favorable. Mortensen (1986) montre que cette stratégie définit un salaire de réserve au-dessus duquel les offres sont toujours acceptées.

De nombreuses contributions théoriques et économétriques ont depuis cherché à déterminer et à estimer les variables influençant ce salaire de réserve. Dès les premiers travaux, l'axe principal de recherche concerne l'impact des mécanismes assurantiels sur l'offre de travail. En offrant au chômeur une source de financement, les mécanismes d'assurance lui permettent d'être plus sélectif et de chercher plus longtemps l'offre d'emploi la plus avantageuse. Cependant, ces études ont en commun de ne se concentrer que sur l'impact de l'assurance publique, à l'instar des estimations de modèles structurels par Lancaster et Chesher (1983) et Narendranathan et al. (1985). Cette approche se trouve élargie dans cet article, par l'intégration des effets de l'assurance privée que constitue l'épargne de précaution.
On estime une équation de salaire de réserve à partir des informations recueillies lors des deux vagues d'enquête du Panel menées en 1994 et 1995 (4). À chaque vague, l'individu interrogé déclare s'il est actuellement chômeur, quel est son salaire de réserve, le montant détenu actuellement en produits d'épargne et s'il perçoit ou non une allocation. Le salaire de réserve déclaré à chaque vague d'enquête est expliqué par le niveau d'épargne déclaré à la même vague.

Les informations relatives au salaire de réserve horaire sont issues de la réponse à la question : «En tenant compte du nombre d'heures hebdomadaires que vous souhaiteriez travailler, quelle rémunération mensuelle nette accepteriez-vous au minimum ?». Le salaire horaire est reconstitué à partir du nombre d'heures de travail souhaité par les individus. L'exploitation

4. Les déclarations relatives aux actifs financiers détenus en 1996 sont trop parcimonieuses pour que cette année puisse être exploitée.

Encadré 1

\section{SOURCES ET CHAMP}

Les données utilisées dans cet article proviennent de l'échantillon français du Panel européen des ménages d'Eurostat et couvrent trois vagues de 1994 à 1996. Cette enquête longitudinale retrace l'historique d'activité, de revenus et de patrimoine financier d'un peu plus de 7000 ménages français de janvier 1993 à décembre 1996. Les calendriers de revenu et d'activité sont individuels et reconstruits rétrospectivement. En revanche le questionnaire relatif au patrimoine financier concerne le ménage dans son ensemble et fournit le stock d'actifs détenu en fin d'année. Chacune des personnes du ménage a été interrogée une première fois en 1994 sur les calendriers de 1993 et 1994. Puis les personnes ont été réinterrogées chaque année, même en cas de déménagement ou de changement dans la composition du ménage.

Cette enquête fournit en complément des informations précises sur les caractéristiques individuelles telles que : l'âge, le sexe, la nationalité, le niveau du diplôme et les conditions de sortie de l'emploi. Elle donne également des informations sur le montant total des revenus (salaires, allocations chômage, prestations sociales) perçus au cours de chaque année. Elle fournit en outre un calendrier mensuel indiquant si l'agent a perçu au cours du mois une allocation chômage. En revanche, il est impossible de reconstruire à partir de ces informations le montant mensuel des allocations puisque ces dernières sont dégressives.

Le Panel européen des ménages décompose la richesse financière en quatre éléments : livrets d'épargne (livrets $\mathrm{A}, \mathrm{B}$, livrets bancaires, CODEVI, LEP, etc.), épargne logement, valeurs mobilières (actions, obligations et SICAV) et produits d'assurance vie et assimilés (dont le PEP). Ces actifs sont déclarés au niveau du ménage. Les réponses sont échelonnées en 8 tranches : (1) moins de 10000 francs (1524 euros), (2) de 10000 à 30000 francs (1524 à 4573 euros), (3) de 30000 à 50000 francs (4 573 à 7622 euros), (4) de 50000 à 100000 francs (7 622 à 15245 euros), (5) de 100000 à 250000 francs (15 245 à 38112 euros), (6) de 250000 à 750000 francs (38 112 à 114337 euros), (7) de 750000 à 1500 000 francs (114337 à 228674 euros), (8) 1500000 francs et plus (228 674 euros et plus).

À chaque vague, le ménage déclare le montant d'actifs possédé actuellement et celui possédé l'année précédente. On dispose donc de deux sources d'information pour les niveaux d'actifs de 1994 et 1995. En revanche, on n'a qu'une source d'information pour les niveaux de 1993 (l'enquête commence en 1994) et de 1996 (les enquêtes menées à partir de 1996 ne sont pas encore disponibles). Ceci est particulièrement préjudiciable dans ce dernier cas puisque les taux déclarés de détention des actifs sont dérisoires. Cette année d'enquête ne pourra donc pas être utilisée.

Enfin, selon des études comparatives préliminaires de Cases (1996b) et Chambaz et al. (1997), il semble que le Panel européen des ménages fournisse des observations très proches de celles de l'enquête Actifs financiers 1991 ou Budgets des familles 1994. Toutefois ces bases ont toutes en commun de sous-estimer les actifs financiers répertoriés par la Comptabilité nationale. 
directe des réponses à cette question soulève des difficultés quant à la sincérité des déclarations (Feldstein et Poterba, 1984). Cependant, la plupart des études précédentes s'accordent pour considérer ce type de réponse comme une information pertinente sur le comportement d'offre de travail (Ridder et Gorter, 1986; Jones, 1988). En outre, la distribution du salaire de réserve semble cohérente avec la moyenne et la variance observées des salaires en France (cf. tableau 1). En particulier, le salaire de réserve horaire est sensiblement égal au Smic horaire de l'époque.

On a retenu comme actif financier le livret d'épargne, qui semble le plus à même, par sa liquidité, de jouer le rôle d'épargne de précaution contre les risques du marché du travail (cf. encadré 2). Les résultats relatifs aux autres produits financiers figurent néanmoins en annexe. Le montant du livret d'épargne est décomposé en quatre niveaux : du niveau 0 (le chômeur ne détient aucun livret) au niveau 3 (le chômeur détient un compte épargne supérieur à 30000 francs (environ 4600 euros). La décomposition des actifs par tranches est détaillée dans l'encadré 2. Le tableau 2 donne le niveau moyen de livret d'épargne détenu par les ménages qui sont chômeurs et déclarent leur salaire de réserve correspondant lors des vagues d'enquête 1994 ou 1995. Quant aux allocations chômage, il s'agit d'une variable indicatrice de perception d'une allocation durant le mois d'interrogation. On n'utilise pas le montant total des allocations chômage car celui-ci correspond à une somme annuelle qu'il est difficile de mensualiser, en l'absence d'informations supplémentaires, du fait de la dégressivité des allocations.

En complément des variables assurantielles, les variables explicatives incluent les principales

\section{Tableau 1 \\ Statistiques descriptives de la population étudiée}

\begin{tabular}{|l|c|c|}
\hline & Moyenne & Écart-type \\
\hline Femme (en \%) & 60,60 & 0,48 \\
Âge & 36,81 & 9,94 \\
Marié(e) (en \%) & 58,20 & 0,43 \\
Conjoint travaille (en \%) & 50,60 & 0,50 \\
\hline Diplôme (en \%): & 21,3 & 0,40 \\
Collège & 49,6 & 0,50 \\
Baccalauréat & 12,9 & 0,33 \\
Baccalauréat + 2 & 7,0 & 0,25 \\
Supérieur & 6,0 & 0,23 \\
Pas de diplôme & 2,8 & 0,16 \\
Non déclaré & 5,66 & 1,715 \\
\hline Salaire de réserve & & \\
horaire (en euros) & \multicolumn{2}{|c|}{}
\end{tabular}

Lecture: pour la définition et le calcul du salaire de réserve horaire, se reporter au texte. Ce salaire est la moyenne des réponses des individus interrogés aux vagues 1994 et 1995. Les autres caractéristiques individuelles sont celles prises usuellement en compte dans les études sur le salaire de réserve.

Champ: personne au chômage lors des vagues d'enquête de 1994 ou 1995.

Source : Panel européen des ménages, Insee.

Encadré 2

\section{ÉPARGNE DE PRÉCAUTION : QUEL ACTIF FINANCIER RETENIR ?}

Quatre types d'actifs financiers sont répertoriés dans le Panel: les livrets d'épargne, les valeurs mobilières, l'épargne logement, et les produits d' assurance-vie (cf. encadré 1). Chaque actif est classé par tranches de telle sorte qu'il est impossible d'agréger le montant d'actifs total détenu par un chômeur. Au demeurant, une telle agrégation aurait peu de sens dans la mesure où la nature de ces actifs diffère fortement. Seul le livret d' épargne peut véritablement correspondre à une épargne de précaution contre les risques du marché du travail. II est suffisamment liquide pour qu'un ménage puisse puiser dans ce stock afin de financer des périodes de chômage. II constitue une épargne de précaution largement plus répandue que les autres types d'actifs parmi les chômeurs.

Les valeurs mobilières recouvrent certains actifs liquides (actions) et d'autres qui le sont moins telles les obligations qui entraînent des moins-values si elles sont vendues avant maturité. Mais ce type d'actif est très peu répandu dans la population édudiée (cf. annexes).
Quant à l' épargne logement et aux produits d'assurance-vie, ils répondent par définition à des motifs beaucoup plus éloignés des risques du marché du travail et sont en outre très peu liquides.

Cet article se concentre donc essentiellement sur l'assurance fournie par le livret d'épargne. Cependant les régressions relatives aux autres actifs sont reportées en annexes afin de vérifier la robustesse des résultats.

Enfin, les huit tranches supérieures au niveau 2 sont regroupées en un unique niveau afin de comparer des groupes de tailles homogènes. On distingue ainsi quatre niveaux d'épargne : le niveau 0 regroupant les chômeurs qui ne possèdent pas de livret, le niveau bas (niveau 1: $0<$ Livret $\leq 10000$ Frs) $(0<\leq 1520$ euros), le niveau intermédiaire (niveau 2 : 10000 Frs $<$ Livret $\leq 30000$ Frs) (1 520 euros $<\leq 4570$ euros) et le niveau élevé (niveau 3 : Livret > 30000 Frs) (4 570 euros <). 
caractéristiques individuelles usuellement prises en compte pour expliquer le salaire de réserve. Ce dernier est susceptible de croître avec l'âge (représentant l'expérience professionnelle potentielle) et avec le diplôme (qui synthétise les qualifications d'un chômeur). On distingue à cet effet les sans-diplôme, le niveau collège, le niveau baccalauréat, le niveau baccalauréat plus deux années d'études (Deug, IUT, BTS, etc.), le niveau d'études supérieures et enfin les non-déclarés. On tient aussi compte du sexe du chômeur. Les caractéristiques du conjoint éventuel sont également susceptibles d'influer sur le salaire de réserve en offrant une source d'assurance supplémentaire au chômeur. Plusieurs stratégies ont été explorées afin de tenir compte de cette relation. On aurait pu retenir le revenu du conjoint comme variable explicative. Une telle variable aurait recouvert un montant si le conjoint travaille, et une indicatrice (de la perception d'une allocation chômage), s'il est au chômage. Le coefficient associé à cette variable devenait alors difficilement interprétable. Aussi a-t-on retenu les caractéristiques attenantes, à savoir le fait que la personne soit mariée et que son conjoint travaille. Les caractéristiques de la population étudiée au regard de ces variables sont détaillées par le tableau 1. L'individu représentatif de cette population est une femme ayant un niveau d'étude équivalent au baccalauréat, déclarant un salaire horaire de réserve de 37,13 francs (5,66 euros) et possédant un livret d'épargne d'un montant inférieur à 10000 francs (1 524 euros).

En ce qui concerne la variable « livret d'épargne », son impact a été différencié selon

\section{Tableau 2 \\ Répartition de la population par niveau de livret d'épargne}

\begin{tabular}{|l|c|}
\hline $\begin{array}{l}\text { Niveau 0 } \\
\text { (aucun livret) }\end{array}$ & 41,00 \\
\hline $\begin{array}{l}\text { Niveau 1 } \\
\text { (moins de 10 000 francs) } \\
\text { (moins de 1 524 euros) }\end{array}$ & 35,60 \\
\hline $\begin{array}{l}\text { Niveau 2 } \\
\text { (de 10 000 francs à 30 000 francs) } \\
\text { (de 1524 euros à 4 573 euros) }\end{array}$ & 11,70 \\
\hline $\begin{array}{l}\text { Niveau 3 } \\
\text { (plus de 30 000 francs) } \\
\text { (plus de 4 573 euros) }\end{array}$ & 11,70 \\
\hline
\end{tabular}

Lecture : l'épargne considérée concerne l'épargne détenue par le ménage sous forme de livret déclarée lors des vagues d'enquête 1994 ou 1995. Pour plus de précision se reporter au texte et aux encadrés 1 et 2 .

Champ: personne au chômage lors des vagues d'enquête de 1994 ou 1995

Source : Panel européen des ménages, Insee. ses niveaux d'accroissement pour tenir compte de la différence de taille des tranches. On introduit à cet effet des variables indicatrices pour chaque niveau du livret. La variable indicatrice du niveau 1 est égale à un si le chômeur détient ce niveau d'actifs et à zéro autrement, et ainsi de suite. Cette stratégie renseigne sur l'impact d'un accroissement du niveau 0 de référence vers le niveau 1 , ou vers le niveau 2 , ou enfin vers le niveau 3.

\section{Au-dessus de 4600 euros d'épargne, le montant du salaire de réserve augmente avec celui du livret d'épargne}

Les coefficients des caractéristiques individuelles sont significatifs et ont un signe conforme à la théorie économique (cf. tableau 3). Le salaire de réserve est plus élevé pour les chômeurs qui disposent d'un diplôme supérieur au baccalauréat, qui sont mariés et dont le conjoint travaille. Il s'accroît aussi avec l'âge du chômeur mais décroît fortement si cette personne est une femme.

Quant aux mécanismes assurantiels, ils ont tous un impact significativement positif sur le salaire de réserve. Conformément aux prédictions

Tableau 3

Équation du salaire de réserve

\begin{tabular}{|l|c|c|}
\hline \multicolumn{1}{|c|}{ Variable } & Coefficient & Écart-type \\
\hline Livret d'épargne: & Référence & \\
Niveau 0 & 0,011 & 0,019 \\
Niveau 1 & 0,032 & 0,036 \\
Niveau 2 & $0,080^{\star \star}$ & 0,034 \\
Niveau 3 & $0,046^{\star \star}$ & 0,020 \\
\hline Allocation chômage & $0,007^{\star \star}$ & 0,001 \\
Âge & $0,004^{\star}$ & 0,002 \\
Marié & 0,002 & 0,002 \\
Conjoint travaille & $-0,114^{\star \star}$ & 0,020 \\
Femme & & \\
\hline Diplôme : & $-0,128^{\star \star}$ & 0,040 \\
Aucun diplôme & $-0,090^{\star \star}$ & 0,024 \\
Collège & Référence & 0,028 \\
Baccalauréat & $0,138^{\star \star}$ & 0,044 \\
Baccalauréat +2 & $0,266^{\star \star}$ & 0,057 \\
Diplôme supérieur & 0,072 & 0,043 \\
Non déclaré & $3,339^{\star \star}$ & \multicolumn{2}{|c|}{0,244} \\
Constante & \multicolumn{2}{|c|}{778} \\
\hline R2 & \multicolumn{2}{|c|}{} \\
\hline Nombre d'observations & \multicolumn{2}{|c|}{} \\
\hline
\end{tabular}

Lecture : modèle estimé par les moindres carrés ordinaires.

${ }^{\star}$ : significatif au seuil de $10 \%$; ${ }^{*}$ : significatif au seuil de $5 \%$. Pour une définition précise des niveaux du livret d'épargne, se reporter aux encadrés 1 et 2 et au texte.

Champ : chômeurs présents aux enquêtes de 1994 et 1995, et qui appartiennent à un ménage dont la structure est restée stable au cours de cette période.

Source : Panel européen des ménages, Insee. 
théoriques de Danforth (1979), la richesse a toujours un impact positif sur le salaire de réserve des chômeurs. Cependant cette influence n'est statistiquement significative qu'à partir d'un patrimoine de 30000 francs (4 600 euros environ). Ce résultat montre qu'il n'existe pas de relation monotone entre le niveau du salaire de réserve et le niveau de richesse, contrairement aux prédictions théoriques de Danforth (1979). En revanche, le montant à partir duquel l'influence de l'épargne devient significative (30 000 francs) est caractéristique : il correspond approximativement au niveau annuel moyen d'allocations chômage qu'un chômeur perçoit dans la base de données. Ce résultat est riche d'enseignements: l'auto-assurance n'a d'impact sur la stratégie de recherche d'emploi que pour des niveaux équivalents à ceux de l'assurance publique.

En ce qui concerne les autres actifs financiers, tous concourent à l'accroissement du salaire de réserve (cf. annexe 1). Cependant, seule l'influence des valeurs mobilières est significative. Ce résultat est cohérent avec la nature des actifs, l'épargne logement et les produits d'assurance-vie s'inscrivant dans des projets de plus long terme et plus éloignés des risques du marché du travail. De plus, très peu de chômeurs possèdent l'un de ces trois types d'actifs (cf. tableaux descriptifs des actifs en annexe), ce qui limite la pertinence des estimations.

\section{Épargne de précaution et durée de recherche d'emploi}

D ans une seconde étape, on estime l'effet de l'épargne de précaution sur la durée du chômage. Dans le modèle canonique de prospection d'emploi, la probabilité de sortir du chômage se compose de deux termes : la probabilité pour un chômeur de recevoir une offre, puis celle de l'accepter. Or, pour des conditions macro-économiques données, une hausse du salaire de réserve diminue la probabilité d'accepter une offre et rallonge ainsi la durée du chômage. Les modèles théoriques de prospection d'emploi avec accumulation d'actifs prédisent donc une relation induite positive entre la durée du chômage et le niveau d'épargne (deuxième proposition de Danforth, cf. supra). Pour tester la pertinence empirique de cette relation, on estime l'influence du stock d'épargne détenu au début de l'épisode de chômage sur la durée de cet épisode.

\section{Les chômeurs dépourvus d'épargne restent plus longtemps au chômage...}

Cette estimation procède en deux temps. On calcule en premier lieu l'estimateur de KaplanMeier qui donne la probabilité empirique de rester chômeur par groupe d'individus. Cet estimateur est non-paramétrique et constitue uniquement une procédure de comptage des épisodes de chômage selon le groupe d'appartenance. Pour chaque estimation, le niveau d'épargne correspond à celui déclaré lors de la vague d'enquête précédant l'épisode de chômage afin de s'assurer du sens de causalité de l'épargne vers la durée du chômage. On distingue d'abord deux groupes, selon que les agents possèdent ou non un livret d'épargne. Les personnes ne disposant d'aucun stock d'actif restent plus longtemps au chômage que les personnes disposant d'un avoir financier (cf. tableau 4-A). Ainsi, un chômeur ne disposant d'aucun livret a $61 \%$ de chances d'être encore au chômage au bout de 5 mois contre $54 \%$ pour un chômeur détenant un compte épargne. Ce premier résultat, qui est contraire aux prédictions des modèles théoriques, est en revanche conforme à l'étude empirique de Stancanelli (1999) sur la Grande Bretagne. Cette relation est donc robuste et relativise les raccourcis trop hâtifs entre salaire de réserve et durée du chômage.

\section{... mais la durée de chômage est d'autant plus longue que le niveau d'épargne est élevé}

En effet, les chômeurs qui ne détiennent pas de livret ont un salaire de réserve inférieur aux autres. Leur probabilité de refuser une offre d'emploi est donc moindre. Mais encore faut-il que cette population ait la même probabilité de recevoir des offres que les chômeurs possédant un compte épargne. Malheureusement, le Panel européen des ménages ne fournit pas d'information complète sur cette variable. Cependant, les chômeurs qui n'ont pas pu se constituer au préalable un stock d'épargne de précaution sont vraisemblablement le plus souvent d'anciens salariés qui occupaient un poste peu qualifié et trop faiblement rémunéré. Ils possèdent donc des caractéristiques individuelles moins favorables sur le marché du travail.

Cette explication est validée par l'application de l'estimateur de Kaplan-Meier au sous-ensemble des chômeurs possédant un livret d'épargne positif. Dans cette sous-population, la probabilité de 
rester chômeur augmente avec le stock d'épargne. Un chômeur détenant un livret de niveau 3 a $73 \%$ de chances d'être encore au chômage au bout de cinq mois contre $53 \%$ pour un chômeur détenant un livret de niveau 1 (cf. tableau 4-B).

Ainsi les prédictions des modèles théoriques de recherche d'emploi avec épargne de précaution (Hansen et Imrohoroglu, 1992; Wang et Williamson, 2000) doivent-elles être relativisées. La relation croissante entre la richesse et la durée du chômage n'est vérifiée que dans le cas de chômeurs détenant une épargne positive.

\section{Un impact sur le taux de sortie du chômage comparable à celui de l'assurance chômage}

Dans un second temps l'analyse est affinée par l'estimation des effets propres à l'épargne de précaution sur la durée du chômage assortie du contrôle des autres caractéristiques individuelles. Cette estimation est limitée aux chômeurs qui ont déclaré une épargne positive. Dans ce dessein, on estime l'impact du stock d'épargne détenu au début d'un épisode de chômage sur la fonction de hasard. Celle-ci représente le taux instantané de sortie du chômage à la période $t$, sachant que l'individu était chômeur jusqu'alors. On utilise à cet effet un modèle de durée semi-paramétrique à hétérogénéité inobservée (cf. encadré 3).

On se limite pour ces estimations aux chômeurs présents lors des trois vagues d'enquête, afin de pouvoir suivre des trajectoires plus longues. Dans ce sous-ensemble, on ne retient que les chômeurs appartenant à un ménage dont la structure est restée stable au cours de la période, puisque le patrimoine financier est déclaré au niveau du ménage. On prend pour variables explicatives les caractéristiques susceptibles de rendre compte de la probabilité de recevoir une offre d'emploi. Il s'agit en premier lieu de l'âge, qui traduit la plus forte exclusion du marché du travail des plus jeunes et des plus âgés. Un motif similaire (les femmes sont davantage exclues) explique le choix du sexe. On tient également compte du niveau d'étude en distinguant le type de filière suivie : sans diplôme, diplôme général (BEPC, baccalauréat), diplôme technique (CAP, BTS, IUT, écoles techniques) et diplôme supérieur (universitaires et assimilés). Il a semblé préférable de distinguer les diplômes selon la filière et non selon le niveau d'études, contrairement à la perspective adoptée pour l'estimation du salaire de réserve. La filière paraît en effet plus à même de capter la probabilité de recevoir une offre car elle permet de distinguer les différences de conjoncture économique selon les secteurs. Les statistiques descriptives de la population étudiée, suivant ces différentes caractéristiques, figurent dans le tableau 5.

De même que pour l'estimation du salaire de réserve, les mécanismes assurantiels sont captés par les allocations chômage et la détention de différents produits financiers. La variable allocation chômage est une variable indicatrice (variant avec le temps) de perception d'une allocation par le chômeur.

Tableau 4

Probabilité de rester au chômage

\section{A - Selon la détention d'un livret d'épargne}

\begin{tabular}{|c|c|c|}
\hline $\begin{array}{c}\text { Durée } \\
\text { du chômage } \\
\text { (en mois) }\end{array}$ & $\begin{array}{c}\text { Aucun livret } \\
\text { d'épargne }\end{array}$ & Livret d'épargne \\
\hline 1 mois & 0,870 & 0,855 \\
2 mois & 0,765 & 0,735 \\
5 mois & 0,606 & 0,539 \\
10 mois & 0,423 & 0,364 \\
20 mois & 0,296 & 0,192 \\
30 mois & 0,206 & 0,123 \\
\hline
\end{tabular}

\section{B - Selon le niveau du livret d'épargne}

\begin{tabular}{|c|c|c|c|}
\hline $\begin{array}{c}\text { Durée de } \\
\text { chômage } \\
\text { (en mois) }\end{array}$ & $\begin{array}{l}\text { Livret de } \\
\text { niveau 1 }\end{array}$ & $\begin{array}{l}\text { Livret de } \\
\text { niveau 2 }\end{array}$ & $\begin{array}{l}\text { Livret de } \\
\text { niveau 3 }\end{array}$ \\
\hline 1 mois & 0,831 & 0,901 & 0,966 \\
2 mois & 0,698 & 0,791 & 0,812 \\
5 mois & 0,532 & 0,572 & 0,734 \\
10 mois & 0,353 & 0,430 & 0,459 \\
20 mois & 0,189 & 0,198 & 0,383 \\
30 mois & 0,142 & 0,159 & 0,255 \\
\hline
\end{tabular}

Lecture : un individu ne détenant aucun livret d'épargne a près de trois chances sur dix d'être encore au chômage au bout de 20 mois, alors que cette probabilité n'est que d'environ deux chances sur dix s'il est détenteur d'un livret d'épargne (tableau 4-A). Si cet individu détient un livret d'épargne, cette probabilité est sensiblement plus élevée lorsque ce livret est de niveau 3 (près de 4 chances sur dix), que lorsqu'il est d'un niveau plus faible (niveau 2, et a fortiori niveau 1 : environ deux chances sur dix). Pour la définition des niveaux, se reporter au texte et aux encadrés 1 et 2. Ces estimations (probabilités empiriques) sont obtenues au moyen d'un estimateur de KaplanMeier (se reporter au texte). Le niveau d'épargne correspond à celui qui a été déclaré lors de la vague d'enquête précédant l'épisode de chômage.

Champ : chômeurs présents aux enquêtes de 1994, 1995 ou 1996, et qui appartiennent à un ménage dont la structure est restée stable au cours de cette période.

Source : Panel européen des ménages, Insee. 
Quant à la variable épargne, elle correspond au montant du livret d'épargne déclaré lors de l'enquête précédant l'épisode de chômage étudié. Ainsi, à titre d'exemple, les épisodes commencés en 1994 seront expliqués par le stock de richesse déclaré à la fin de l'année 1993. Cette procédure évite tout biais d'endogénéité du niveau de richesse par rapport à la durée du chômage. Elle conduit à éliminer tous les épisodes de chômage commencés avant 1994 puisque l'on ne dispose pas d'information sur le stock d'actifs initial détenu au début de l'année 1993. Les estimations se restreignent donc à la période allant de janvier 1994 à décembre 1996. Le tableau 6 donne le niveau moyen de livret d'épargne détenu avant un épisode de chômage au cours de cette période. Enfin, comme pour le salaire de réserve, l'analyse se limite à l'impact du livret d'épargne sur la durée du chômage, aucun des trois autres types d'actifs (valeurs mobilières, épargne logement et produits d'assurance-vie) ne s'avérant significatif (cf. annexe 2).

La stratégie d'estimation est la même que pour l'équation du salaire de réserve. On distingue donc des niveaux similaires d'accroissement du livret d'épargne. Cependant, le niveau de référence est maintenant le niveau 1 , les chômeurs ne possédant pas de livret ayant été exclus. On estime alors l'impact d'un accroissement sur la fonction de hasard selon que cet accroissement consiste en un passage du niveau 1 de référence vers le niveau 2 ou vers le niveau 3 .

Les résultats de ces estimations figurent dans le tableau 7. Un signe négatif (positif) indique que la caractéristique individuelle associée tend à faire diminuer (augmenter) le taux de sortie du chômage. Les coefficients associés aux niveaux

\section{Encadré 3}

\section{LE MODĖLE DE DURÉE SEMI-PARAMÉTRIQUE À HÉTÉROGÉNÉITÉ INOBSERVÉE}

Le modèle de durée utilisé ici s'inscrit dans la famille des modèles à hasard proportionnel où un hasard « de base » est déplacé par le vecteur des caractéristiques individuelles. Le taux de hasard d'un individu de caractéristiques $X_{i}$ peut s'écrire:

$$
\lambda_{i}\left(t / X_{i}, \varepsilon_{i}\right)=\varepsilon_{i} \lambda_{0}(t) \exp \left(X_{i}^{\prime} \beta\right)
$$

Le paramètre $\lambda_{0}(t)$ correspond à la probabilité de base d'un individu de sortir du chômage lorsque ses caractéristiques individuelles sont ignorées. Le terme $\exp \left(X_{i}^{\prime} \beta\right)$ mesure comment les caractéristiques individuelles accroissent ou réduisent le hasard de base, $\beta$ représentant le vecteur des paramètres à estimer. Enfin, le paramètre multiplicatif $\varepsilon_{i}$ est destiné à capter I'hétérogénéité inobservée qui n'est pas mesurée par le vecteur de variables exogènes $X_{i}$. En effet, il est fort probable que de nombreuses variables (telles que le fait d'avoir reçu et refusé des offres) sont inconnues, bien qu'elles influencent le processus de sortie du chômage. Or l'omission de cette hétérogénéité inobservée peut entraîner un biais négatif (dit du « moverstayer ») dans l'estimation du paramètre de dépendance temporelle.

On suppose que ce paramètre suit une loi Gamma de moyenne 1 et de variance $\sigma^{2}$. Seule la variance du paramètre d'hétérogénéité est estimée. Le test de présence d'hétérogénéité inobservée revient à tester $\sigma^{2}=0$. Si $\sigma^{2}$ est significativement différent de zéro, alors on peut conclure à la présence d'une hétérogénéité inobservée influençant les sorties du chômage.

Le calendrier d'activité étant mensuel, on adopte une approche semi-paramétrique dans la lignée de Meyer (1990). Cette approche revient à estimer directement le hasard de base pour chaque intervalle de temps plutôt que de lui imposer une loi a priori afin d'éviter tout biais de spécification. Le hasard de base s'écrit alors :

$\lambda_{0}(t)=1-\exp \left(-\exp \left(\alpha_{1} \gamma_{1}+\ldots+\alpha_{L} \gamma_{L}\right)\right)$

où $L$ représente le nombre d'intervalles considérés, et où la variable $\gamma_{i}$ est une variable dichotomique égale à 1 si $t$ appartient au $i$ intervalle, et à 0 sinon.

Par souci de simplicité, une formulation constante par segment est retenue pour le hasard de base. On suppose qu'il est constant sur des intervalles de deux mois au début (du premier au $26^{\mathrm{e}}$ mois). Ces intervalles sont représentés par les dummies de $\gamma_{1}$ à $\gamma_{12}$. Puis la probabilité empirique de sortir du chômage étant plus stable du $26^{\mathrm{e}}$ mois au $38^{\mathrm{e}}$ mois, on fait l'hypothèse que le hasard de base est constant sur deux intervalles de six mois représentés par les variables $\gamma_{13}$ et $\gamma_{14}$.

En définitive, la Log-vraissemblance estimée s'écrit (Jenkins, 1997) :

$$
\begin{aligned}
& \mathrm{LL}=\sum_{N} \log \left(\left(1-d_{i}\right) A_{i}+d_{i} B_{i}\right) \\
& \text { avec }: A_{i}=\left[1+\sigma^{2} \sum_{j=1}^{t i} \exp \left(x_{i}^{\prime} \beta+\gamma_{j}\right)\right]^{-\frac{1}{\sigma^{2}}} \\
& B_{i}= \begin{cases}{\left[\begin{array}{ll}
1+\sigma^{2} \sum_{j=1}^{t i-1} \exp \left(x_{i}^{\prime} \beta+\gamma_{j}\right) \\
1-A_{i} & \text { si } t_{i}=1
\end{array}\right.}\end{cases}
\end{aligned}
$$


du livret d'épargne sont tous négatifs. Ce signe est cohérent avec l'estimation du salaire de réserve : il confirme que la détention d'un livret d'épargne joue à la baisse sur la probabilité de sortir du chômage. De plus, cette baisse est d'autant plus accentuée que le montant de l'épargne correspondante est élevé, comme le montrent les coefficients respectifs des indicatrices d'un passage du niveau 1 au niveau 2 , et du niveau 1 au niveau 3. Cependant, cette influence n'est statistiquement significative qu'à partir d'un saut du niveau 1 vers le niveau 3 , la probabilité de sortir

Tableau 5

Statistiques descriptives de la sous-population étudiée

\begin{tabular}{|c|c|c|}
\hline & Moyenne & Écart-type \\
\hline $\begin{array}{l}\text { Durée moyenne de chômage } \\
\text { (en mois) } \\
\text { Inscription à l'ANPE (en \%) } \\
\text { Femme (en \%) } \\
\text { Âge à la fin de l'épisode } \\
\text { de chômage }\end{array}$ & $\begin{array}{l}7,42 \\
89,6 \\
58,9 \\
38,8\end{array}$ & $\begin{array}{r}7,487 \\
0,301 \\
0,500 \\
10,393\end{array}$ \\
\hline \begin{tabular}{|l} 
Diplôme (en \%): \\
Aucun diplôme \\
Diplôme général \\
Diplôme technique \\
Diplôme du supérieur
\end{tabular} & $\begin{array}{r}2,1 \\
39,0 \\
43,9 \\
15,0\end{array}$ & $\begin{array}{l}0,134 \\
0,487 \\
0,496 \\
0,357\end{array}$ \\
\hline $\begin{array}{l}\text { Personnes ayant connu au moins } \\
\text { Nombre } \\
\text { Part dans l'échantillon (en \%) }\end{array}$ & épisode de & $\begin{array}{l}\text { hômage : } \\
3\end{array}$ \\
\hline Taux de chômage moyen (en \%) & & \\
\hline
\end{tabular}

Lecture : les caractéristiques individuelles prises en compte sont celles susceptibles de jouer sur la probabilité de recevoir une offre d'emploi.

Champ: chômeurs présents aux enquêtes de 1994, 1995 ou 1996, et qui appartiennent à un ménage dont la structure est restée stable au cours de cette période.

Source : Panel européen des ménages, Insee.

Tableau 6

Répartition de la sous-population étudiée par niveau de livret d'épargne

\begin{tabular}{|c|c|}
\hline $\begin{array}{l}\text { Niveau } 0 \\
\text { (aucun livret) }\end{array}$ & 49,86 \\
\hline $\begin{array}{l}\text { Niveau } 1 \\
\text { (moins de } 10000 \text { francs) } \\
\text { (moins de } 1524 \text { euros) }\end{array}$ & 26,94 \\
\hline $\begin{array}{l}\text { Niveau } 2 \\
\text { (de } 10000 \text { francs à } 30000 \text { francs) } \\
\text { (de } 1524 \text { euros à } 4573 \text { euros) }\end{array}$ & 12,39 \\
\hline $\begin{array}{l}\text { Niveau } 3 \\
\text { (plus de } 30000 \text { francs) } \\
\text { (plus de } 4573 \text { euros) }\end{array}$ & 10,81 \\
\hline
\end{tabular}

Lecture : l'épargne considérée concerne l'épargne détenue par le ménage sous forme de livret. Pour plus de précision se reporter au texte et aux encadrés 1 et 2. Le niveau d'épargne correspond à celui qui a été déclaré lors de la vague d'enquête précédant l'épisode de chômage.

Champ: chômeurs présents aux enquêtes de 1994, 1995 ou 1996, et qui appartiennent à un ménage dont la structure est restée stable au cours de cette période.

Source : Panel européen des ménages, Insee. du chômage diminuant alors de $23,1 \%$ (5). Ce chiffre est proche de celui associé à l'impact des allocations chômage : la perception d'une allocation diminue de $26,9 \%$ la probabilité de sortir du chômage au cours d'un mois.

L'épargne de précaution sous forme de livret affecte donc positivement la durée du chômage, mais uniquement à partir d'un niveau d'actifs supérieur à 30000 francs (4 573,47 euros). Mais dans ce cas, l'impact de l'épargne est sensiblement de même ampleur que celui de la perception des allocations chômage. Ce résultat se comprend mieux lorsque l'on se réfère au montant annuel minimal d'allocations que perçoivent les chômeurs éligibles dans la base de données et qui est de l'ordre de 30000 francs. Ainsi les effets des deux types de mécanismes assurantiels sont-ils proches pour des sommes monétaires de même ordre de grandeur. Ce résultat est cohérent avec les estimations de l'influence de l'épargne de précaution sur le salaire de réserve.

Tableau 7

Estimation du taux de sortie du chômage

\begin{tabular}{|c|c|c|}
\hline Variables & Coefficient & Écart-type \\
\hline $\begin{array}{l}\text { Femme } \\
\text { Âge } \\
\text { Diplôme : } \\
\text { Aucun diplôme } \\
\text { Diplôme Général } \\
\text { Diplôme technique } \\
\text { Diplôme supérieur }\end{array}$ & $\begin{array}{c}-0,458^{\star \star} \\
-0,020^{\star \star} \\
\text { - 0,571 } \\
\text { Référence } \\
0,014^{\star \star} \\
0,011^{\star \star}\end{array}$ & $\begin{array}{l}0,096 \\
0,005 \\
0,073 \\
\\
0,003 \\
0,004\end{array}$ \\
\hline $\begin{array}{l}\text { Allocation Chômage } \\
\text { Livret d'épargne : } \\
\text { Niveau } 1 \\
\text { Niveau } 2 \\
\text { Niveau } 3\end{array}$ & $\begin{array}{l}-0,313^{\star \star} \\
\text { Référence } \\
-0,163 \\
-0,263^{\star \star}\end{array}$ & $\begin{array}{l}0,117 \\
0,082\end{array}$ \\
\hline Constante & $-0,867^{\star \star}$ & 0,227 \\
\hline$\sigma^{2}$ & $4,996 \mathrm{E}-6$ & $4,274 \mathrm{E}-6$ \\
\hline Log-vraisemblance & \multicolumn{2}{|c|}{$-1421,19$} \\
\hline $\begin{array}{l}\text { Nombre de durées men- } \\
\text { suelles de chômages } \\
\text { observées }\end{array}$ & \multicolumn{2}{|c|}{4717} \\
\hline
\end{tabular}

Lecture : estimation par un modèle de durée semi-paramétrique à hétérogénéité inobservée (se reporter à l'encadré 3).

* : significatif au seuil de $10 \%$; ** : significatif au seuil de $5 \%$. Le niveau d'épargne correspond à celui qui a été déclaré lors de la vague d'enquête précédant l'épisode de chômage.

Champ: chômeurs présents aux enquêtes de 1994, 1995 ou 1996, et qui appartiennent à un ménage dont la structure est restée stable au cours de cette période.

Source : Panel européen des ménages, Insee.

5. Dans le cadre des modèles à hasard proportionnel, les coefficients estimés mesurent l'impact multiplicatif de chaque variable sur le ratio du hasard de base. La forme exponentielle nécessite une transformation afin de pouvoir quantifier l'impact des caractéristiques individuelles. Ainsi, le chiffre de 23,1\% à été calcule comme suit. L'accroissement du niveau 1 au niveau 3 multiplie le hasard de base par $\exp (-0,263)=0,769$. II diminue alors de $23,1 \%(=1-0,769)$ le taux de hasard de base par rapport à une personne dont la caractéristique financière est ignorée. 
Enfin, les caractéristiques individuelles conditionnant la probabilité de recevoir une offre d'emploi exercent toutes une influence significative et conforme à l'intuition. Le fait d'être une femme, ou plus âgé (caractéristiques de l'individu à la fin de la période de chômage), semblent réduire considérablement les chances de sortie du chômage. En revanche, cette probabilité augmente considérablement avec le niveau de diplôme : les diplômés de l'enseignement technique et supérieur ont ainsi plus de chances de retrouver un emploi que ceux de l'enseignement général.

\section{Les chômeurs accepteraient plus facilement les offres d'emploi au moment de la réduction de leur allocation}

Le hasard de base estimé (à savoir, la probabilité de sortie du chômage) est une fonction globalement décroissante du temps passé au chômage (cf. graphique). Cette relation négative n'a rien de surprenant: l'employabilité ou le capital humain d'un chômeur diminuant progressivement avec le temps passé au chômage, sa probabilité de sortir du chômage s'amenuise en proportion. Cependant cette décroissance n'est pas monotone puisque le taux de hasard augmente de façon ponctuelle au bout d'un an, d'un an et demi puis de deux ans. Cette évolution est semblable à ce que l'on a constaté récemment au niveau France entière (Dormont et al., 2001). Ces pics ponctuels doivent être reliés à la dégressivité par paliers du niveau des allocations chômage en France. Le système de l'allocation unique dégressive (AUD) inciterait les chômeurs à accepter plus facilement les offres d'emploi juste avant chaque réduction de ressources. Cette évolution par à-coups du taux de hasard souligne la nécessité d'estimer directement le hasard de base plutôt que de lui imposer une spécification a priori. Quant à la variance du terme d'hétérogénéité inobservée, elle n'est pas significative, ce qui suggère que l'hétérogénéité individuelle est déjà captée par les variables exogènes du modèle.

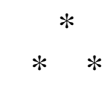

Le Panel européen des ménages, en réunissant des sources d'information relatives à la fois au patrimoine financier et aux calendriers d'activité, permet de valider empiriquement certains résultats théoriques relatifs à l'impact de l'épargne de précaution sur les comportements de recherche d'emploi : tout d'abord l'effet positif et significatif du stock d'épargne sur cette variable, puis la croissance du temps passé au chômage avec le niveau du stock d'épargne détenu au début de l'épisode de chômage.

Cependant, l'importance de l'épargne de précaution dans le comportement de recherche d'emploi des chômeurs doit être relativisée à l'aune de deux résultats. D'une part, ce type d'assurance individuelle n'a des effets significatifs qu'à partir de niveaux élevés et comparables aux revenus annuels procurés par les allocations chômage. D'autre part, la relation positive entre le niveau d'épargne et la durée du chômage ne se vérifie que pour ceux des chômeurs qui détiennent des actifs. Ces résultats

\section{Probabilité conditionnelle de sortie du chômage selon l'ancienneté}

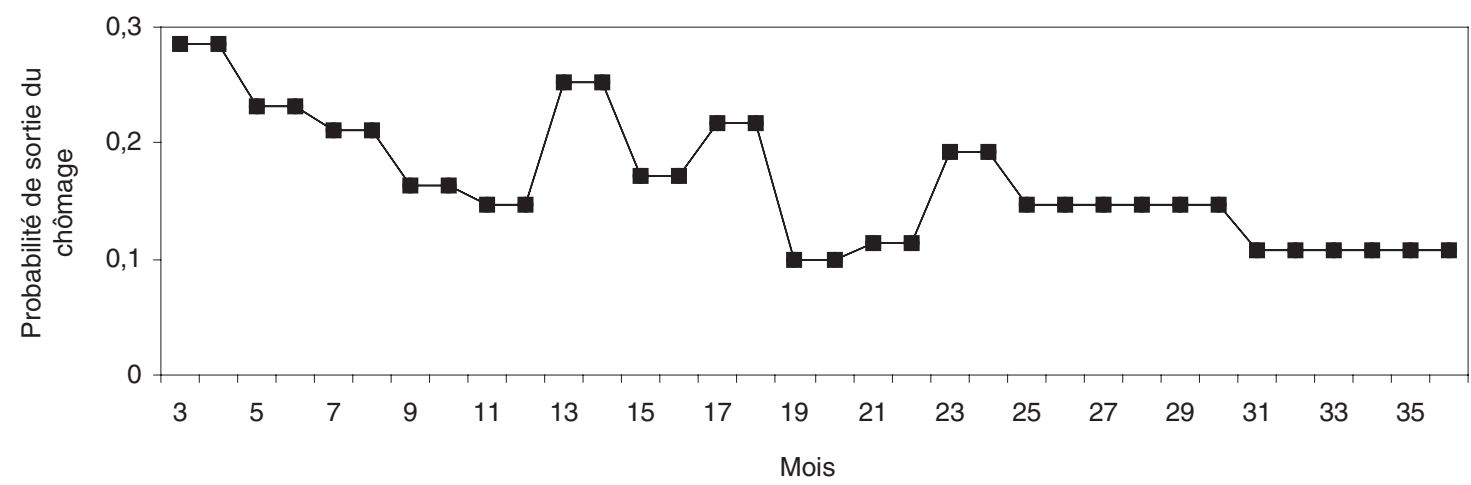

Lecture : un chômeur ayant 19 mois d'ancienneté a 10 chances sur 100 de retrouver un emploi.

Champ : chômeurs présents aux enquêtes de 1994, 1995 ou 1996, et qui appartiennent à un ménage dont la structure est restée stable au cours de cette période.

Source : Panel européen des ménages, Insee. 
établissent un lien entre les caractéristiques institutionnelles du marché du travail, et le comportement d'épargne des chômeurs. En effet, le niveau de l'épargne de précaution est susceptible d'être d'autant plus important que l'assurance publique contre les risques de chômage est faible. Cela suggère de comparer l'impact de l'épargne sur le comportement de recherche d'emploi entre des pays aux systèmes d'assurance chômage différents : offrant une base de données européenne, le Panel européen des ménages rend possible une telle étude comparative.
Enfin, si l'épargne de précaution partage les vertus assurantielles des allocations chômage, elle n'en constitue pas pour autant un substitut. En effet, les ménages les plus sujets au risque de chômage n'ont pas les moyens de se constituer une épargne de précaution, ce qu'atteste la plus longue durée passée au chômage des personnes dépourvues d'épargne. La substitution d'un système d'assurance sociale par un système d'auto-assurance conduirait alors à un accroissement conséquent des inégalités, ce que suggérait l'approche théorique (Algan et Allais, 2001).

Les auteurs remercient l'Insee pour l'accès au fichier français du Panel européen des ménages ainsi que Pascale Breuil-Genier, Emmanuel Duguet, Jean-Olivier Hairault, François Langot et deux rapporteurs anonymes pour leurs recommandations et suggestions.

\section{BIBLIOGRAPHIE}

Algan Y. et Allais O. (2001), « The Comparative Stabilyzing Effects of Self-Insurance and Social Insurance », document de travail, EUREQua.

Algan Y., Chéron A., Hairault J.-O. et Langot F. (2000), «Épargne de précaution et chômage : une évaluation quantitative de l'auto-assurance », Cahiers de la MSE, n 2000.92.

Acemoglu D. et Shimer R. (1999), «Efficient Unemployment Insurance », Journal of Political Economy, vol. 107, n 5, pp. 893-929.

Cases C. (1996a), « Assurance chômage et offre de travail », Économie et Statistique, n 291-292, pp. 139-148.

Cases C. (1996b), « Méthodologie du Panel européen des ménages : exploitation de la vague $1 \mathrm{du}$ fichier français », document de travail, Insee.

Chambaz C., Saunier J.-M. et Valdelièvre H. (1997), «Méthodologie du Panel européen des ménages : exploitation de la vague 2 du fichier français », document de travail, Insee.

Danforth J.-P. (1979), « On the Role of Consumption and Decreasing Absolute Risk Aversion in Job Search Theories », in Studies in the Economics of Search, Lippman et McCall (eds), NorthHolland.

Dormont B. Fougère D. et Prieto A. (2001), «L'effet de l'allocation unique dégressive sur la reprise d'emploi », Économie et Statistique, n 343 , pp. 3-28.

Feldstein M. et Poterba J. (1984), «Unemployment Insurance and Reservation Wage », Journal of Public Economics, vol. 23, pp. 141-167.

Hansen G.-D. et Imrohoroglu A. (1992), « The Role of Unemployment Insurance in an Economy with Liquidity Constraints and Moral Hazard», Journal of Political Economy, vol. 100, $\mathrm{n}^{\circ} 1$, pp. 118-142.

Jenkins S.-P. (1997), « Discrete Time Proportional Hazard Regression », Stata Technical Bulletin, n 39 , pp. 22-32.

Joseph G. et Weitzemblum T. (2000), «Recherche d'emploi et épargne de précaution : dynamique transitoire v.s équilibre de long terme », document de travail, EUREQua.

Jones S. (1988), «The Relationship between Unemployment Spells and Reservation Wages as a Test of Search Theory », The Quarterly Journal of Economics, vol. 103, n ${ }^{\circ}$, pp. 741-765.

Lancaster T. et Chesher A. (1983), « An Econometric Analysis of Reservation Wages », Econometrica, vol. 51, pp. 1661-1676.

McCall J.-J. (1970), « Economics of Information and Job Search », Quarterly journal of Economics, vol. 84 (1), pp. 113-126. 
Meyer B.-B. (1990), « Unemployment Insurance and Unemployment Spells », Econometrica, vol. 58 , pp. 757-782.

Mortensen D.-T. (1986), « Job Search and Labor Market Analysis », in Handbook of Labour Economics, vol. 2.

Narendranathan W., Nickell S. et Stern J. (1985), «Unemployment Benefits Revisited», Economic Journal, vol. 95, pp. 307-329.

Ridder G. et Gorter K. (1986), « Unemployment Benefits and the Search Behaviour : an Empirical
Investigation », mimeo, Cornell University and University of Amsterdam.

Stancanelli E. (1999), « Do the Rich Stay Unemployed Longer ? An Empirical Study for the UK», Oxford Bulletin of Economics and Statistics, vol. 61(3).

Villieu P. (1997), Macroéconomie : consommation et épargne, coll. Repères, La Découverte.

Wang C. et Williamson S.-D. (2000), « Moral Hazard, Optimal Unemployment Insurance and Experience Rating », document de travail, Carnergie Mellon University. 


\section{IMPACT DES AUTRES ACTIFS FINANCIERS SUR LE SALAIRE DE RÉSERVE}

Tableau A

Répartition de la population par niveau et par type d'actifs

En \%

\begin{tabular}{|l|c|c|c|}
\hline & Valeurs mobilières & Épargne logement & Assurance-vie \\
\hline $\begin{array}{l}\text { Niveau 0 } \\
\text { (aucun actif) }\end{array}$ & 85,9 & 72,8 & 74,9 \\
\hline $\begin{array}{l}\text { Niveau 1 } \\
\text { (moins de 10 000 francs) } \\
\text { (moins de 1 524 euros) }\end{array}$ & 3,0 & 7,9 & 8,4 \\
\hline $\begin{array}{l}\text { Niveau 2 } \\
\text { (de 10 000 francs à 30 000 francs) } \\
\text { (de 1 524 euros à 4 573 euros) }\end{array}$ & 3,1 & 8,0 & 7,7 \\
\hline $\begin{array}{l}\text { Niveau 3 } \\
\text { (plus de 30 000 francs) } \\
\text { (plus de 4 573 euros) }\end{array}$ & & & \\
\hline
\end{tabular}

Lecture : on considère ici l'épargne détenue sous une autre forme que le livret, le niveau étant celui déclaré lors de l'enquête en même temps que le salaire de réserve. Se reporter au texte et aux encadrés 1 et 2.

Champ : chômeurs présents aux enquêtes de 1994, 1995 ou 1996, et qui appartiennent à un ménage dont la structure est restée stable au cours de cette période.

Source : Panel européen des ménages, Insee.

Tableau B

\section{Équation du salaire de réserve}



Lecture : * : significatif au seuil de $10 \%$; ** : significatif au seuil de $5 \%$. Pour chaque variable, on a fait figurer l'écart-type entre parenthèse sous le coefficient correspondant. Le niveau d'épargne correspond à celui qui a été déclaré lors de la vague d'enquête.

Champ : chômeurs présents aux enquêtes de 1994, 1995 ou 1996, et qui appartiennent à un ménage dont la structure est restée stable au cours de cette période.

Source : Panel européen des ménages, Insee. 


\section{IMPACT DES AUTRES ACTIFS FINANCIERS SUR LA DURÉE DU CHÔMAGE}

Tableau A

Répartition de la population par niveau et par type d'épargne

En \%

\begin{tabular}{|l|c|c|c|}
\hline & Valeurs mobilières & Épargne logement & Assurance-vie \\
\hline $\begin{array}{l}\text { Niveau 0 } \\
\text { (aucun actif) }\end{array}$ & 85,4 & 72,2 & 73,5 \\
\hline $\begin{array}{l}\text { Niveau 1 } \\
\text { (moins de 10 000 francs) } \\
\text { (moins de 1 524 euros) }\end{array}$ & 2,3 & 7,5 & 8,0 \\
\hline $\begin{array}{l}\text { Niveau 2 } \\
\text { (de 10 000 francs à 30 000 francs) } \\
\text { (de 1 524 euros à 4 573 euros) }\end{array}$ & 4,4 & 9,0 & 8,2 \\
\hline $\begin{array}{l}\text { Niveau 3 } \\
\text { (plus de 30 000 francs) } \\
\text { (plus de 4 573 euros) }\end{array}$ & & & 11,3 \\
\hline
\end{tabular}

Lecture : pour chacun des types d'actif, le niveau correspond au stock détenu au début de l'épisode de chômage. Pour plus de précision se reporter au texte et aux encadrés 1 et 2.

Champ : chômeurs présents aux enquêtes de 1994, 1995 ou 1996, et qui appartiennent à un ménage dont la structure est restée stable au cours de cette période.

Source : Panel européen des ménages, Insee.

Tableau B

\section{Estimation du taux de sortie du chômage}

\begin{tabular}{|c|c|c|c|}
\hline & Valeurs mobilières & Épargne logement & Assurance-vie \\
\hline $\begin{array}{l}\text { Femme } \\
\text { Âge } \\
\text { Diplôme : } \\
\text { Aucun diplôme } \\
\text { Diplôme général } \\
\text { Diplôme technique } \\
\text { Diplôme supérieur }\end{array}$ & $\begin{array}{c}-0,309^{\star \star} \\
(0,133) \\
-0,041^{\star \star} \\
(0,005) \\
\\
-0,797^{\star \star} \\
(0,273) \\
\text { Référence } \\
0,023^{\star \star} \\
(0,009) \\
0,016^{\star \star} \\
(0,002)\end{array}$ & $\begin{array}{c}-0,193^{\star \star} \\
(0,033) \\
-0,023^{\star \star} \\
(0,010) \\
\\
-0,622^{\star \star} \\
(0,173) \\
\text { Référence } \\
0,019^{\star \star} \\
(0,005) \\
0,022^{\star \star} \\
(0,009)\end{array}$ & $\begin{array}{c}-0,501^{\star \star} \\
(0,150) \\
-0,027^{\star \star} \\
(0,008) \\
\\
-0,474^{\star \star} \\
(0,073) \\
\text { Référence } \\
0,026^{\star *} \\
(0,111) \\
0,011^{\star \star} \\
(0,003)\end{array}$ \\
\hline $\begin{array}{l}\text { Allocation chômage } \\
\text { Actifs financiers }\end{array}$ & $\begin{array}{c}-0,208^{* *} \\
(0,089) \\
-0,073 \\
(0,079)\end{array}$ & $\begin{array}{c}-0,363^{\star \star} \\
(0,134) \\
-0,060 \\
(0,153)\end{array}$ & $\begin{array}{c}-0,480^{\star *} \\
(0,155) \\
-0,013 \\
(0,091)\end{array}$ \\
\hline Constante & $\begin{array}{r}-0,180 \\
(0,176)\end{array}$ & $\begin{array}{r}-0,750^{*} \\
(0,424)\end{array}$ & $\begin{array}{r}-0,468^{*} \\
(0,224)\end{array}$ \\
\hline$\sigma^{2}$ & $\begin{array}{l}1,586 \mathrm{E}-4 \\
(2,248 \mathrm{E}-4)\end{array}$ & $\begin{array}{l}1,234 \mathrm{E}-4 \\
(1,987 \mathrm{E}-4)\end{array}$ & $\begin{array}{l}1,324 \mathrm{E}-5 \\
(1,465 \mathrm{E}-5)\end{array}$ \\
\hline Log-vraisemblance & $-299,39$ & $-750,408$ & $-604,658$ \\
\hline $\begin{array}{l}\text { Nombre de durées mensuelles } \\
\text { de chômage observées }\end{array}$ & 1353 & 2583 & 2460 \\
\hline
\end{tabular}

Lecture : ** : significatif au seuil de $5 \%$. Estimation par un modèle de durée semi-paramétrique à hétérogénéité inobservée (se reporter à l'encadré 3). Les variables démographiques et d'éducation ont pour niveau de référence le niveau de diplôme général. Pour chaque variable explicative, on a fait figurer l'écart-type entre parenthèse sous le coefficient correspondant. Pour le choix et la définition des types d'actifs, on se reportera aux encadrés 1 et 2 , et au texte. Le niveau d'épargne correspond à celui qui a été déclaré lors de la vague d'enquête précédant l'épisode de chômage.

Champ : chômeurs présents aux enquêtes de 1994, 1995 ou 1996, et qui appartiennent à un ménage dont la structure est restée stable au cours de cette période.

Source : Panel européen des ménages, Insee. 